\title{
Brain Regions Related to Impulsivity Mediate the Effects of Early Adversity on Antisocial Behavior
}

\author{
Scott Mackey, Bader Chaarani, Kees-Jan Kan, Philip A. Spechler, Catherine Orr, \\ Tobias Banaschewski, Gareth Barker, Arun L.W. Bokde, Uli Bromberg, Christian Büchel, \\ Anna Cattrell, Patricia J. Conrod, Sylvane Desrivières, Herta Flor, Vincent Frouin, \\ Jürgen Gallinat, Penny Gowland, Andreas Heinz, Bernd Ittermann, Marie-Laure Paillère \\ Martinot, Eric Artiges, Frauke Nees, Dimitri Papadopoulos-Orfanos, Luise Poustka, \\ Michael N. Smolka, Sarah Jurk, Henrik Walter, Robert Whelan, Gunter Schumann, \\ Robert R. Althoff, Hugh Garavan, and The IMAGEN Consortium
}

\begin{abstract}
BACKGROUND: Individual differences in impulsivity and early adversity are known to be strong predictors of adolescent antisocial behavior. However, the neurobiological bases of impulsivity and their relation to antisocial behavior and adversity are poorly understood.

METHODS: Impulsivity was estimated with a temporal discounting task. Voxel-based morphometry was used to determine the brain structural correlates of temporal discounting in a large cohort $(n=1830)$ of 14 - to 15-year-old children. Mediation analysis was then used to determine whether the volumes of brain regions associated with temporal discounting mediate the relation between adverse life events (e.g., family conflict, serious accidents) and antisocial behaviors (e.g., precocious sexual activity, bullying, illicit substance use).

RESULTS: Greater temporal discounting (more impulsivity) was associated with 1) lower volume in frontomedial cortex and bilateral insula and 2) greater volume in a subcortical region encompassing the ventral striatum, hypothalamus and anterior thalamus. The volume ratio between these cortical and subcortical regions was found to partially mediate the relation between adverse life events and antisocial behavior.

CONCLUSIONS: Temporal discounting is related to regions of the brain involved in reward processing and interoception. The results support a developmental imbalance model of impulsivity and are consistent with the idea that negative environmental factors can alter the developing brain in ways that promote antisocial behavior.
\end{abstract}

Keywords: Adversity, Antisocial behavior, Development, Impulsivity, Temporal discounting, Voxel-based morphometry

http://dx.doi.org/10.1016/j.biopsych.2015.12.027

Substantial public resources have been allocated to fund education and health initiatives aimed at improving the living conditions of disadvantaged children on the basis of the idea that child development is a sensitive window of opportunity during which the foundations of lifelong well-being are established (1). This policy perspective is supported by a wealth of research indicating that adverse events in the formative years of childhood have long-term consequences in terms of physical and mental health (2-4). However, the biological bases of these effects, particularly those relating to brain development, are as yet incompletely understood.

Among the known consequences of developmental exposure to adversity is a significant increase in the likelihood of engaging in antisocial behavior (ASB) (5). ASB is defined as risky behavior that does not conform to age-specific standards of conduct. During adolescence, this includes precocious sexual activity, alcohol and drug use, trouble at school, and violent aggression. A central feature of ASB is high trait impulsivity, an association that has been observed with a broad range of measures including laboratory tests such as the temporal discounting task [e.g., (6)] and independent ratings by teachers and parents (7). Moreover, as impulsivity declines with age from adolescence to early adulthood $(8,9)$, the prevalence of ASB also decreases (10). Here, we asked whether brain regions associated with impulsivity could mediate the relation between early adversity and ASB.

Developmental imbalance models explain the declining rate of impulsivity from adolescence to early adulthood in terms of a temporal gap in the growth trajectories of competing 
neurobiological systems [e.g., $(11,12)]$. In the adult brain, the cortex, in particular the frontal cortex, regulates appetitive approach behaviors mediated by subcortical structures to adaptively align behavior with the long-term goals of the individual. Since the cortex develops more slowly than other brain regions, a process that continues well into the third decade of life $(13,14)$, the absence of mature cortical regulation during adolescence may result in unconstrained impulsive behavior $(9,15)$.

Impulsivity is a complex construct that incorporates multiple aspects of decision making, including attention, motor responding, and choice (16). In the present study, impulsivity was assessed by the temporal discounting task, which estimates the rate at which the subjective value of an outcome is reduced as the time to delivery is delayed (17). Low temporal discounting (i.e., less impulsivity) demonstrates an ability to delay immediate gratification in exchange for larger later rewards. Temporal discounting has been shown to have trait-like stability (6), and high discounting rates (i.e., greater impulsivity) have been linked to several problem behaviors, such as substance use and addiction (18-20), pathological gambling (21), poor health and financial decision making (22), and antisocial personality disorder $(23,24)$. The preference in each of these examples for the immediate reinforcing outcome of the problem behavior despite the potential for larger delayed negative consequences (e.g., to health, professional, and social well-being) suggests that temporal discounting may be a transdisease process $(22,25)$. Consequently, clarification of the brain substrates involved in temporal discounting could impact the understanding of several psychiatric disorders characterized by poor impulse control.

In the present study, the structural correlates of temporal discounting were identified by voxel-based morphometry in a large cohort of European children. Mediation analysis was then used to determine whether these brain regions mediate the relation between adverse events and ASB. Cortical and subcortical regions of interest (ROls) associated with temporal discounting were tested as mediators separately. To test the developmental imbalance theory of adolescent impulsivity directly, the ratio between the cortical and subcortical ROI volumes was also calculated and used as a mediator in an additional mediation model.

\section{METHODS AND MATERIALS}

\section{Standard Operating Procedures}

Standard operating procedures for the IMAGEN project are available online at http://www.imagen-europe.com/en/Pub lications_and_SOP.php. All aspects of the IMAGEN project were performed in compliance with the Declaration of Helsinki.

\section{Participants}

A large sample of healthy adolescents was recruited from four European countries (26). A structural magnetic resonance imaging (MRI) brain scan and a temporal discounting score were available in 1830 individuals (51.5\% female; $10.3 \%$ lefthanded; average age 14.55 years). Physical pubertal status was assessed by the Puberty Development Scale (27), and IQ was assessed by the Wechsler Intelligence Scale for Children, 4th edition (28). The Vocabulary and Similarities subscales of the Wechsler Intelligence Scale for Children, 4th edition, were used to produce a measure of verbal IQ, while the Block Design, Matrix Reasoning, and Digit Span subscales were used to produce a measure of (nonverbal) performance IQ. Verbal and performance IQ scores were adjusted to reflect the age of the participants.

\section{Adverse Life Events and Antisocial Behavior Scores}

Negative life events and antisocial behaviors were surveyed by the Life Events Questionnaire (LEQ) adapted from Newcomb et al. (29) and the European School Survey Project on Alcohol and Drugs (ESPAD) $(30,31)$. The ESPAD interview includes items adapted from the Revised Olweus Bully/Victim Questionnaire (32) and the Youth Risk Behavior Survey (33), which assess involvement in bullying as perpetrator and/or victim. Explanatory comments on the relevant subscales within the LEQ and ESPAD and example items are provided in Supplement 1. The scales were selected to capture a broad range of negative events and behaviors. In 14-year-olds, this includes precocious sexual behavior, use of illicit substances (namely alcohol, cigarettes, and other psychoactive drugs), bullying, and running away from home. The Accident/Illness, Family/Parents, and Relocation subscales of the LEQ, as well as the Victim of Bullying subscale of the ESPAD, were individually $Z$-score transformed and then summed to produce a composite measure of adverse life events. Similarly, the Deviance, Sexuality, and Distress subscales of the LEQ, as well as the Perpetrator of Bullying and Lifetime Frequency of Illicit Substance Use subscales of the ESPAD, were individually Z-score transformed and then summed to produce a composite measure of antisocial behavior. Since adolescents frequently explore the boundaries of the acceptable (i.e., all children misbehave; some do so more than others), the ASB score is a continuous measure of age-specific rule breaking and does not attempt to set a threshold between normal and abnormal levels of antisocial behavior.

\section{Estimation of Temporal Discounting}

The computer-administered version of Kirby's Monetary Choice Questionnaire (MCQ) is an efficient and reliable laboratory measure of temporal discounting $(6,34)$. It contains 27 items probing the subject's preference for a range of small immediate rewards versus larger delayed rewards. For example, item 5 asks: "Would you prefer $€ 14$ today or $€ 25$ in 19 days?" Answers were self-paced and made by clicking on one of two digital response buttons with a computer mouse. The range of delays and euro amounts of the rewards across items of the MCQ were selected to represent nine well-spaced levels of temporal discounting as fitted by a hyperbolic function: $V=A(1+k D)$, where $\mathrm{V}$ is the current subjective value of the delayed reward, $A$ is the absolute value of the delayed reward, $D$ is the length of delay in days, and $\mathrm{k}$ is a constant representing the magnitude of the discounting function (35). Higher values of $\mathrm{k}$ indicate greater preference for small immediate rewards and higher impulsivity. Although the rewards were hypothetical, previous research indicates that the use of real rewards does not produce a significantly different pattern of results $(36,37)$. 
The MCQ was scored as described in Kirby et al. (38). That is, $k$ is estimated as the geometric mean of the highest value of $\mathrm{k}$ for which the subject preferred the small immediate reward and the lowest level of $k$ for which the subject preferred the larger delayed reward. If the subject endorsed a preference for only the immediate or only the delayed rewards, then the subject was rated at the highest or lowest of the nine k-estimate levels on the MCQ, respectively. The relatively few subjects who did not endorse a discrete indifference point were excluded from further analysis $(n=14)$.

To test reward magnitude effects on temporal discounting, the nine impulsivity levels on the MCQ are reproduced at three different ranges of approximate reward magnitude (i.e., delayed rewards were offered in small $€ 25-35$, medium $€ 50-60$, and large $€ 75-85$ ranges). A k-estimate is produced for each category of reward magnitude. In preparation for regression on brain volume, a single k-estimate per subject was produced by calculating the geometric mean of the three reward categories. The subjects' unique k-estimates were then approximately normalized by logarithmic transformation.

\section{Acquisition of Anatomical Magnetic Resonance Images}

Details of the MRI acquisition protocols and quality controls have been provided elsewhere (26). Briefly, high-resolution in vivo structural magnetic resonance images were acquired, including a three-dimensional T1-weighted scan based on the Alzheimer's Disease Neuroimaging Initiative protocols (http://adni.loni.usc.edu/methods/documents/mri-protocols/). To accommodate for effects related to imaging site, location was included as a nuisance covariate in statistical analyses.

\section{Voxel-Based Morphometry}

T1-weighted images were processed using the SPM8 (Wellcome Trust Centre for Neuroimaging, London, United Kingdom) (http://www.fil.ion.ucl.ac.uk/spm/software/spm8/) VBM toolbox (Structural Brain Mapping Group, University of Jena, Jena, Germany) (http://dbm.neuro.uni-jena.de/vbm/) with default parameters incorporating the DARTEL toolbox implemented in MATLAB 7.0 (The MathWorks, Inc., Natick, Massachusetts). The standard optimized method of iterative tissue segmentation and spatial normalization using both linear (12-parameter affine) and nonlinear transformations was performed $(39,40)$. More precise details are provided in Supplemental Results. To make the residuals in later analyses conform more closely to a Gaussian distribution and to account for individual differences in brain anatomy, the modulated gray matter images in Montreal Neurological Institute space were smoothed with an isotropic Gaussian kernel of $8 \mathrm{~mm}$ full-width at half maximum. The dependent measure in the subsequent regression analysis was absolute gray matter volume.

\section{Brain Volume Regression for Temporal Discounting}

A whole-brain voxelwise regression of temporal discounting on regional gray matter volume was performed with the $3 \mathrm{dttest}++$ function from the Analysis of Functional Neuroimages (AFNI) software library (National Institute of Mental
Health, Bethesda, MD) (41). Age, sex, handedness, MRI site, puberty status, verbal $I Q$, performance $I Q$, and intracranial volume (ICV) were included as covariates. ROls related to temporal discounting were identified in the resulting statistical parametric map. To control for multiple comparisons, the AFNI Monte Carlo simulation function, 3dClustSim, was used to determine that a cluster containing more than 688 adjacent voxels $(2322 \mu \mathrm{L})$ each significantly correlated with temporal discounting at a voxelwise threshold of $p=.01(t=2.58)$ would have an a posteriori probability of $p<.01$. Average gray matter volume within the four ROls identified by the regression was extracted from each participant and used in subsequent analyses. Since the volumes of the three cortical ROls were highly intercorrelated, these were summed to produce a single cortical volume measure per subject. To assess a developmental imbalance between cortical and subcortical maturation, a ratio of cortical to subcortical volume was calculated by dividing the combined volume of the cortical ROls by the volume of the subcortical ROI. Thus, higher ratio values indicate greater cortical volume relative to the subcortical volume. In SPSS (Version 21.0; IBM Corp., Armonk, NY), partial correlations between the volumes of the brain ROls, the adversity score, and the ASB score were examined while controlling for age, sex, handedness, MRI site, puberty status, verbal IQ, and performance IQ.

\section{Mediation Analysis}

Mediation analysis was performed on data from 1741 participants (51.4\% female; $89.7 \%$ right-handed) in which all mediation variables were available: the adverse life events and antisocial behavior composite scores and the brain volume ROls associated with temporal discounting. The demographic characteristics of these 1741 children were not significantly different than the total group enrolled (Table 1). The mediation analysis was performed using PROCESS, an SPSS macro developed by Andrew Hayes (http://www.processmacro.org/) (42). PROCESS uses an ordinary least squares path analytic framework to estimate direct and indirect mediation effects. The significance of indirect effects is assessed by bootstrap confidence intervals (Cls). Covariates used in the brain volume regression with the exception of ICV were included in the mediation analysis. ICV was not included because it was highly collinear with the volumes of the cortical and subcortical ROls. A separate analysis showed that ICV does not mediate the relationship between adversity and ASB (Supplemental Results). All paths are reported as unstandardized ordinary least squares regression coefficients.

Table 1. Demographic Characteristics of $n=1741$ Participants (51.4\% Female; $89.7 \%$ Right-handed) for Whom All Mediation Variables and Covariates Were Available

\begin{tabular}{lccc}
\hline & Mean (SE) & Median & Range \\
\hline Age (Years) & $14.5(0.01)$ & 14.5 & $12.9-16.1$ \\
\hline Puberty Status & $3.6(0.02)$ & 4 & $1-5$ \\
\hline Performance IQ & $107.6(0.3)$ & 106 & $63-149$ \\
\hline Verbal IQ & $110.8(0.3)$ & 111 & $50-152$ \\
\hline Adversity Score & $0(0.06)$ & -0.21 & $-5.2-11.0$ \\
\hline ASB Score & $0(0.07)$ & -0.38 & $-6.5-16.1$
\end{tabular}

ASB, antisocial behavior. 
Table 2. Partial Correlations Controlling for Sex, Age, Handedness, MRI Site, Performance IQ, Verbal IQ, and Puberty Status

\begin{tabular}{lcrrr}
\hline & Adversity & ASB & Ratio & Cortical Volume \\
\hline Adversity & & & & \\
\hline ASB & $0.357^{a}$ & & & \\
\hline $\begin{array}{l}\text { Cortical/Subcortical } \\
\text { Ratio }\end{array}$ & -0.041 & $-0.090^{a}$ & & \\
\hline Cortical Volume & $-0.053^{b}$ & $-0.089^{a}$ & $0.579^{a}$ & \\
\hline Subcortical Volume & -0.003 & 0.007 & $-0.530^{a}$ & $0.373^{a}$ \\
\hline
\end{tabular}

Ratio, ratio of the combined volume of cortical ROls to the volume of the subcortical ROI. ASB, antisocial behavior; MRI, magnetic resonance imaging; $\mathrm{ROI}$, region of interest.

${ }^{a} p<.01$

${ }^{b} p<.05$.

\section{RESULTS}

\section{Behavior}

Subjects endorsed a wide range of discounting rates on the MCQ. The median k-estimate was 0.013 , which corresponds approximately to being indifferent to the choice between $38 €$ now or $50 €$ in 24 days. Since k-estimates in the three reward categories of the temporal discounting task were highly correlated (small-large $r=.80$; medium-large $r=.84$; smallmedium $r=.83 ; p<.001$ ), a single average k-estimate per subject based on the geometric mean was used in the subsequent brain volume regression (see Supplemental Results for relation of temporal discounting to other laboratory measures of impulsivity). All children reported exposure to at least one of the negative life experiences included in the adversity composite score (e.g., parents' divorce, serious accident or illness in the family, severe bullying) (Supplemental Table S1 and Figure S1). All children also reported at least some antisocial behavior (Supplemental Table S2 and Figure S2). Temporal discounting was correlated with ASB $(r=.144, p<.001)$ but not adversity $(r=.024$, $p=.318)$. Partial correlations between the variables of interest are reported in Table 2. Notably, the adversity score was a significant predictor of ASB $(r=.357, p<.01)$.

\section{Temporal Discounting Correlates With Brain Volume \\ A whole-brain voxelwise regression of temporal discounting on regional gray matter volume identified four significant regions (Figure 1; Table 3). Three cortical ROIs, the frontome- dial cortex and the insula bilaterally, exhibited greater volume in individuals with lower impulsivity. A large subcortical cluster that included the ventral striatum, dorsal hypothalamus, and anterior thalamus exhibited the opposite relation, i.e., greater volume in those with greater impulsivity. The volumes of the brain ROls were not related to reward magnitude in the temporal discounting task (Supplement).}

\section{Mediation Analysis}

To evaluate the significance of the neuroimaging results within the context of real-world events, mediation analysis was used to test the hypothesis that childhood adversity can change brain development in a way that increases ASB (Figure 2). The reported coefficients are unstandardized. After partialing out the effects of the covariates, adverse life events were significantly related to ASB alone (dashed line) and also when the ratio of cortical to subcortical volume was included as a mediator (solid line). The indirect or mediated effect was calculated as the product of the $a$ and $b$ path coefficients. In the PROCESS analysis toolbox, a significant indirect effect is indicated when the bootstrap $\mathrm{Cl}$ does not include zero. There was a significant positive indirect effect of adverse life events on ASB through the ratio of subcortical to cortical volume (indirect effect $=0.0038, \mathrm{SE}=0.0027,95 \% \mathrm{Cl}=0.0001$ to 0.0111). It should be noted that the popular but statistically suboptimal causal steps method developed by Baron and Kenny (43), which infers mediation on the basis of a set of hypothesis tests, was not used in this study. Since the indirect effect itself was assessed with bootstrapped Cls, the signifi-
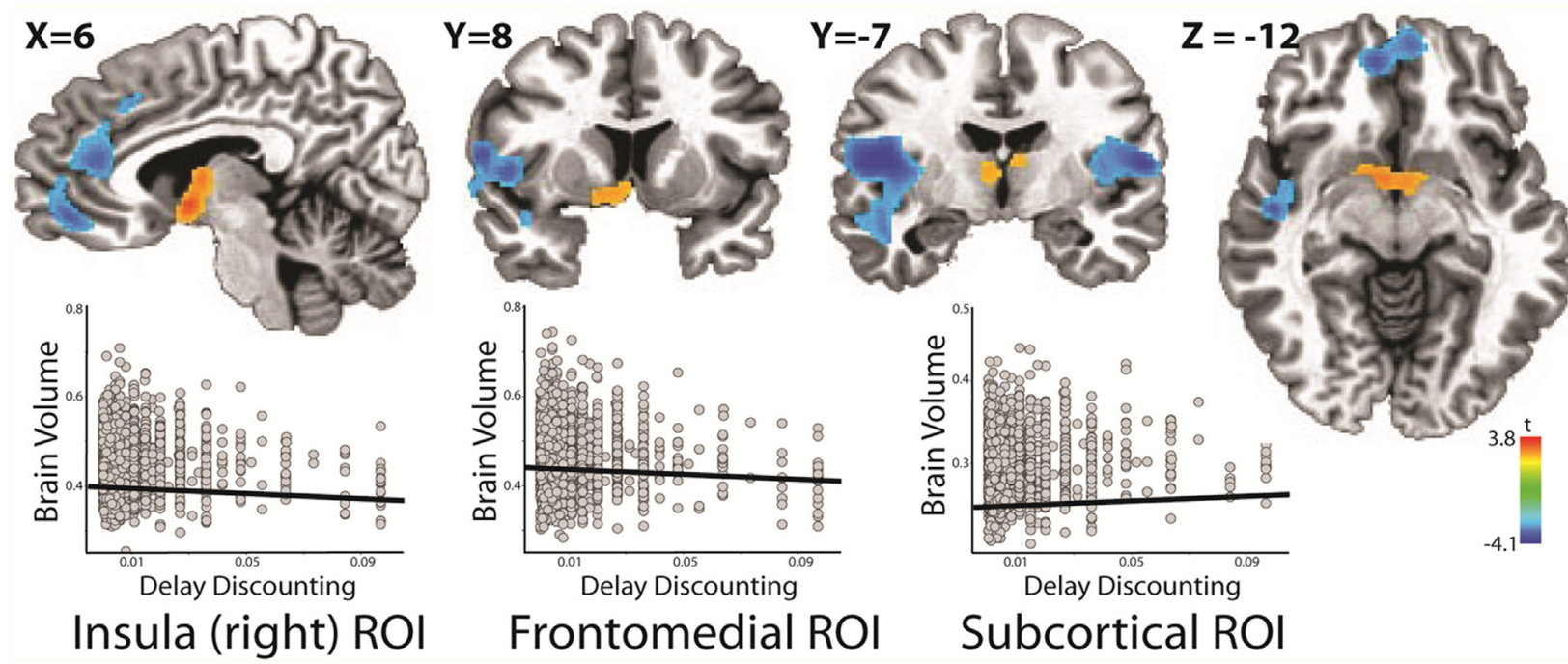

Figure 1. Top row: Regions associated with temporal discounting by brain volume. Warm and cool colors represent more and less volume, respectively. Bottom row: Scatter plots of brain volume versus temporal discounting score in three regions of interest (ROI). 
Table 3. Size and Stereotactic Location of Brain Regions Significantly Associated by Volume With Temporal Discounting

\begin{tabular}{lcrrr}
\hline Region & Cluster Size (Voxels) & \multicolumn{1}{c}{$x$} & \multicolumn{1}{c}{$y$} & $\mathrm{z}$ \\
\hline Right Insula & 3185 & 48 & -8 & 12 \\
\hline Bilateral Frontomedial Cortex & 1996 & -11 & 52 & 12 \\
\hline Left Insula & 1529 & -48 & -14 & 16 \\
\hline Bilateral Subcortical Region & 825 & 3 & 0 & -2 \\
\hline
\end{tabular}

Coordinates $(x, y, z)$ are in Montreal Neurological Institute space.

cance of the individual coefficients of the a $(p=.086)$ and $b(p=.001)$ pathways in the model are not relevant to the interpretation of the indirect effect [a well-developed explanation of this issue is presented in Hayes (42)]. Multiple factors contribute to brain volume, some in opposing directions. Unknown or unaccounted factors that have not been expressly included in the mediation model can obscure the apparent relation between adversity and volume. Nevertheless, the mediation is revealed by testing the indirect effect. Also, since hypothesis testing is fallible, every additional significance test (i.e., tests of $a$ and $b$ pathways) increases the likelihood that a true effect will be mistakenly overlooked. Simulation research indicates the bootstrap method is more robust to nonnormality and has better type I error control than the causal steps method and the Sobel test (42).

Further analyses examining the mediating effects of cortical and subcortical volumes separately indicated that there was a
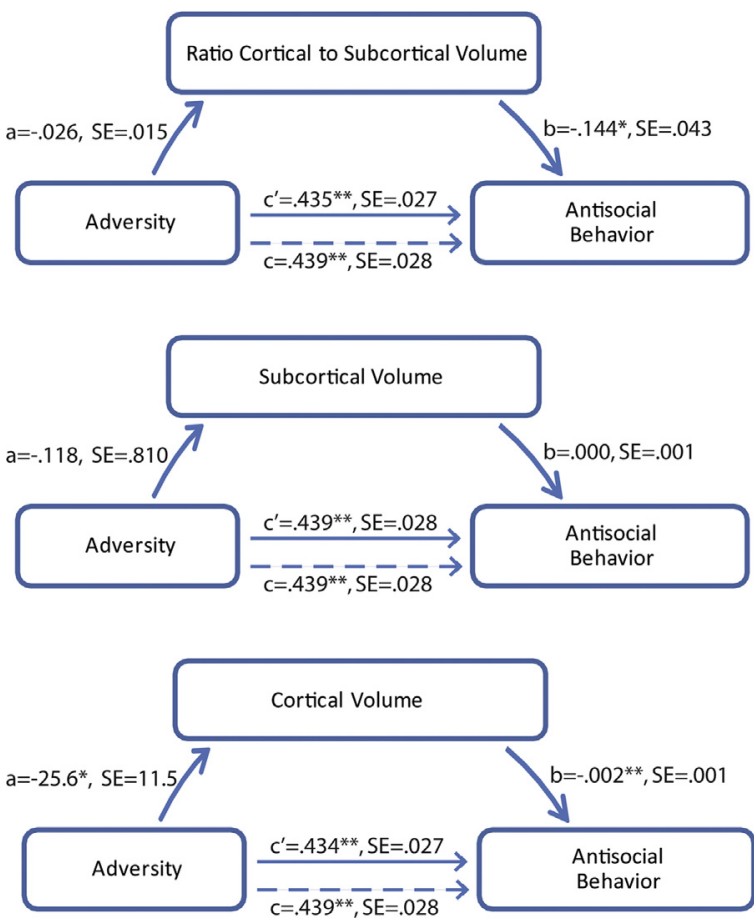

Figure 2. Three mediation models of the relationships between adversity, impulsive antisocial behavior, and gray matter volume in brain regions associated with temporal discounting. Dotted line denotes the effect of adversity on antisocial behavior when the mediating variable is not included. All paths are reported as unstandardized ordinary least squares regression coefficients. ${ }^{*} p<.05,{ }^{\star *} p<.005$. SE, standard error. significant indirect effect through cortical volume alone (indirect effect $=0.005, \mathrm{SE}=0.003, \mathrm{Cl}=0.001$ to 0.013 ) but not subcortical volume alone (indirect effect $=0.000, \mathrm{SE}=0.001$, $\mathrm{Cl}=-0.002$ to 0.001 ) (Figure 2). The ROI volumes were not merely a substitute for temporal discounting because temporal discounting did not on its own significantly mediate the relationship between adversity and ASB (indirect effect $=$ $0.004, \mathrm{SE}=0.027, \mathrm{Cl}=-0.003$ to 0.013 ).

\section{DISCUSSION}

The present study showed that greater impulsivity on a temporal discounting task is associated with lower gray matter volume in the frontomedial and insular cortex and greater gray matter volume in a subcortical region encompassing the ventral striatum, hypothalamus, and anterior thalamus (Figure 1; Table 3). In addition, the relation between adverse life events and ASB was partially mediated by the gray matter volume ratio between the subcortical and cortical regions identified by the task (Figure 2). A mediation model with cortical volume alone as mediator suggests that the indirect effect may be driven predominantly by individual differences in the cortex. These results are consistent with developmental imbalance theories of impulsivity and with the idea that negative environmental factors can alter the developing brain in ways that promote problematic behavior.

The association of this specific set of brain regions with temporal discounting draws considerable support from previous research in humans and animals (44-47). The subcortical region identified includes the ventral striatum, which receives projections from midbrain dopamine cells signaling the presence or expectation of reward. The frontomedial region identified includes portions of the dorsomedial and ventromedial prefrontal cortex. This cortical region encodes the subjective value of future rewards during decision making (48). Individuals with lesions in the ventromedial prefrontal cortex make disadvantageous choices on gambling tasks that model real-life decisions and gauge the extent to which the individual is able to disregard large short-term wins and losses to make larger longer term gains, an insensitivity for future consequences that has been called myopia for the future (49). The insula is located at the center of a network of brain structures involved in the perception and regulation of the internal state of the body (50). It has been proposed that the physiological state of the body plays a central role in emotion and motivation. Damage to the insula has also been linked to deficits in decision making (51).

A recent functional neuroimaging study reported that the age-related decline in temporal discounting from adolescence to early adulthood is associated with changes in the magnitude of neural activity in a set of brain regions closely matching the present findings, including the frontomedial cortex, ventral striatum, and insula (44). Moreover, more impulsive individuals exhibited greater activity in the ventral striatum and less activity in the frontomedial cortex, a finding that mirrors the differential volumetric findings in the present study. Only a handful of studies have used structural neuroimaging to search for brain correlates of temporal discounting (52-54). These studies found that greater impulsivity was significantly correlated with striatal and frontal cortical gray 
matter volume in partial agreement with the present findings. White matter differences related to temporal discounting have also been reported in the frontal and temporal lobes $(55,56)$.

The current study's results support a developmental imbalance model of impulsivity according to which heightened impulsivity during adolescence is a consequence of the differential growth trajectories of competing brain systems involved in choice behavior $(9,11,12,15,25)$. Approach behavior is mediated by the mesolimbic dopamine system, which includes the ventral striatum and is integrated with the longterm goals of the individual by input from the cortex. The slower development of the cortex $(13,14)$ produces an imbalance during adolescence favoring immediate gratification at the expense of larger delayed rewards. Consistent with the imbalance model, the current study found that parts of the cortex (i.e., frontomedial cortex and insula) are smaller and a subcortical region encompassing the ventral striatum is larger in adolescents who are more impulsive. Due to the crosssectional nature of the present study, future longitudinal studies will be required to address whether cortical and subcortical maturation has differently timed periods of greater sensitivity to negative environmental factors and whether the structural differences observed in this study are long lasting or can be altered by future positive environmental factors.

Adversity during development produces a wide range of negative physical and psychiatric problems that may manifest only later in life (2-4). A large number of studies have established that many brain systems in humans and animal models are harmed by early adversity (57-59). A few recent neuroimaging studies, like the current one, have begun to search for specific brain mediators of the negative psychiatric effects produced by adversity. For example, Gorka et al. (60) report that frontomedial and hippocampal cortical volumes mediate the relation between childhood mistreatment and trait anxiety, while Rao et al. (61) have shown that hippocampal volume mediates the relation between childhood mistreatment and higher risk of depression in adulthood (61). An advantage of the current study was that the brain areas examined were identified by an independent measure of impulsivity rather than by their direct association with adversity. This is also the first study to look for brain mediators of early adversity on ASB.

The interpretation of this study is limited by several factors. ASB is a complex behavior to which many environmental factors likely contribute, including factors not considered here, such as modeling of peer behavior, opportunity, and sensation seeking $(5,15)$. For most of the population, the occurrence of ASB peaks in adolescence when situational ASB is common, even normative, then declines as the individual enters early adulthood (10). Like ASB, impulsivity is also a multifactorial construct that likely depends on multiple brain structures $(16,62,63)$. Separate investigations would be required to examine the potential contribution of these other types of impulsivity to ASB. Another potential limitation is that the LEQ and ESPAD do not provide information about the extent to which events used to construct the adversity score might have been influenced by each child's behavior (e.g., serious accident or illness in the family, relocation, parental divorce, or victim of bullying all might have been influenced to a greater or lesser degree by the behavior of the individual child). The interpretation of the adversity score as a measure of negative life events experienced by the child should be qualified by the understanding that children interact with their environment and are not merely passive objects in it. In addition, the large sample size increases the likelihood of detecting small effects, which is an advantage of the present study given the inherent noisiness of behavioral and neuroimaging data. It is also possible that individual temporal discounting rates could have been more precisely assessed with other instruments [e.g., $(24,64)]$. Despite these constraints, the results fit well with developmental imbalance theories of adolescent impulsivity and indicate that significant negative life events may alter the developing brain in ways that promote ASB.

\section{ACKNOWLEDGMENTS AND DISCLOSURES}

This work received support from the European Union-funded FP6 Integrated Project IMAGEN (Grant No. LSHM-CT- 2007-037286), the FP7 project IMAGEMEND and the Innovative Medicine Initiative Project EU-AIMS (Grant No. 115300-2), the National Institute on Drug Abuse (Grant No. 1R21DA03838), the National Institutes of Health (Grant No. 1P20GM103644-01A1 awarded to the Vermont Center on Behavior and Health), a Medical Research Council Programme Grant (Grant No. 93558), the Swedish funding agency FORMAS, the Wellcome Trust (University of Cambridge), the National Institute for Health Research Biomedical Research Centre at South London and Maudsley National Health Service Foundation Trust and King's College London, the Department of Health United Kingdom, the Bundesministerium für Bildung und Forschung (Grant Nos. 01GS08152; 01EV0711; eMED Grant No. SysAlc01ZX1311A; Forschungsnetz AERIAL), and the Fondation de France.

Members of the IMAGEN Consortium not listed as individual authors include: Karl Mann, Maren Struve, Marcella Rietschel, Rainer Spanagel, Mira Fauth-Bühler, Sabina Millenet, and Yvonne Grimmer at the Central Institute of Mental Health, University of Heidelberg; Nikolay Ivanov, Nicole Strache, Michael Rapp, Andreas Ströhle, and Jan Reuter at Charité, Universitätsmedizin Berlin; Alexis Barbot, Benjamin Thyreau, Yannick Schwartz, and Christophe Lalanne at the Comissariat à l'Energie Atomique; Jean-Luc Martinot, Zuleima Bricaud, Fanny Gollier Briand, Hervé Lemaitre, Jessica Massicotte, Helene Vulser, Jani Pentillä, and André Galinowski at the Institut national de la santé et de la recherche médicale; Tianye Jia, Helen Werts, Lauren Topper, Laurence Reed, Chris Andrew, Catherine Mallik, Barbara Ruggeri, Charlotte Nymberg, Lindsay Smith, Eva Loth, Stephanie Havatzias, Kerstin Stueber, and Argyris Stringaris at the Institute of Psychiatry, King's College London; Patrick Constant at PERTIMM (Asnières-Sur-Seine); Ruediger Brühl, Albrecht Ihlenfeld, and Bernadeta Walaszek at the Physikalisch-Technische Bundesanstalt; Thomas Hübner, Kathrin Müller, Stephan Ripke, Sarah Rodehacke, Eva Mennigen, Dirk Schmidt, Nora Vetter, and Veronika Ziesch at the Technische Universität Dresden; Jennifer Jones at the University College Dublin; Jean-Baptiste Poline at the University of California, Berkeley; Tahmine Fadai, Juliana Yacubian, and Sophia Schneider at the University of Hamburg; Claire Lawrence, Craig Newman, Kay Head, and Nadja Heym at the University of Nottingham; and Tomáš Paus, Zdenka Pausova, and Amir Tahmasebi at the University of Toronto.

Dr. Banaschewski served in an advisory or consultancy role for Actelion, Hexal Pharma, Lilly, Medice, Novartis, Oxford outcomes, PCM scientific, Shire, and Viforpharma. He received conference support or speaker's fee by Janssen McNeil, Lilly, Medice, Novartis, and Shire. He has been involved in clinical trials conducted by Shire \& Viforpharma. The present work is unrelated to these grants and relationships. Dr. Gallinat has received research funding from AstraZeneca and speaker fees from Janssen-Cilag and Otsuka. Dr. Paillère Martinot received compensation from JanssenCilag for continuing medical education related activities. Dr. Poustka has received conference attendance support or received speaking fees from Eli Lilly, Medice, Novartis, and Shire. Dr. Robert R. Althoff receives grant or research support from the National Institute of Mental Health, the National 
Institute of General Medical Sciences, and the Klingenstein Third Generation Foundation. He receives honoraria from continuing medical education presentations for Oakstone Medical Publishing and the Massachusetts General Hospital Psychiatry Academy and is employed, in part, by the nonprofit Research Center for Children, Youth, and Families that has developed and publishes the Achenbach System of Empirically-Based Assessment. The remaining authors reported no biomedical financial interests or potential conflicts of interest.

\section{ARTICLE INFORMATION}

From the Department of Psychiatry (SM, BC, K-JK, PS, CO, RRA, HG), University of Vermont, Burlington, Vermont; Department of Child and Adolescent Psychiatry and Psychotherapy (TB, LP), Central Institute of Mental Health, Medical Faculty Mannheim, Heidelberg University, Mannheim, Germany; Centre for Neuroimaging Sciences (GB), Institute of Psychiatry, Psychology and Neuroscience, King's College London, London, United Kingdom; Discipline of Psychiatry (ALWB), School of Medicine and Trinity College Institute of Neurosciences, Trinity College Dublin, Dublin, Ireland; University Medical Centre Hamburg-Eppendorf (UB), Hamburg, Germany; Medical Research Council Social, Genetic and Developmental Psychiatry Centre (CB, SD, GS), Institute of Psychiatry, Psychology and Neuroscience, King's College London, London, United Kingdom; Department of Psychiatry (AC, PJC), Universite de Montreal, CHU Ste Justine Hospital, Montreal, Canada; Department of Psychological Medicine and Psychiatry (PJC), Institute of Psychiatry, Psychology and Neuroscience, King's College London, London, United Kingdom; Department of Cognitive and Clinical Neuroscience (HF, FN), Central Institute of Mental Health, Medical Faculty Mannheim, Heidelberg University, Mannheim, Germany; Neurospin (VF, DP-O), Commissariat à l'Energie Atomique, CEA-Saclay Center, Paris, France; Department of Psychiatry and Psychotherapy (JG), University Medical Centre Hamburg-Eppendorf, Hamburg, Germany; Sir Peter Mansfield Imaging Centre School of Physics and Astronomy (PG), University of Nottingham, University Park, Nottingham, United Kingdom; Department of Psychiatry and Psychotherapy (AH, HW), Campus Charité Mitte, Charité, Universitätsmedizin Berlin; Physikalisch-Technische Bundesanstalt (BI), Berlin, Germany; Institut National de la Santé et de la Recherche Médicale (M-LPM), Unit 1000 Neuroimaging and Psychiatry, University Paris Sud, University Paris Descartes-Sorbonne Paris Cité; Department of Adolescent Psychopathology and Medicine, Maison de Solenn, Cochin Hospital, Paris; Institut National de la Santé et de la Recherche Médicale (EA), Unit 1000 Neuroimaging and Psychiatry, University Paris Sud, University Paris Descartes-Sorbonne Paris Cité; Psychiatry Department, Orsay Hospital, Orsay, France; Department of Child and Adolescent Psychiatry and Psychotherapy (LP), Medical University of Vienna, Vienna, Austria; Department of Psychiatry and Neuroimaging Center (MNS, SJ), Technische Universität Dresden, Dresden, Germany; Department of Psychology (RW), University College Dublin, Dublin, Ireland; and Department of Biological Psychology (K-JK), VU University, Amsterdam, Netherlands.

Address correspondence to Scott Mackey, Ph.D., University of Vermont, Psychiatry, Nerve Lab, Suite 1400 Fletcher Allen Health Care-UHC Campus, 1 South Prospect Street, Burlington, VT 05401; E-mail: msmackey@uvm.edu.

Received Jul 13, 2015; revised Dec 21, 2015; accepted Dec 28, 2015.

Supplementary material cited in this article is available online at http:// dx.doi.org/10.1016/j.biopsych.2015.12.027.

\section{REFERENCES}

1. Center on the Developing Child (2010): The foundations of lifelong health are built in early childhood. Available at: http://developingchild. harvard.edu/wp-content/uploads/2010/05/Foundations-of-LifelongHealth.pdf. Accessed June 1, 2015.

2. Anda RF, Felitti VJ, Bremner JD, Walker JD, Whitfield C, Perry BD, et al. (2006): The enduring effects of abuse and related adverse experiences in childhood. A convergence of evidence from neurobiology and epidemiology. Eur Arch Psychiatry Clin Neurosci 256: 174-186.
3. Gilbert LK, Breiding MJ, Merrick MT, Thompson WW, Ford DC Dhingra SS, Parks SE (2015): Childhood adversity and adult chronic disease: An update from ten states and the District of Columbia, 2010. Am J Prev Med 48:345-349.

4. Norman RE, Byambaa M, De R, Butchart A, Scott J, Vos T (2012): The long-term health consequences of child physical abuse, emotional abuse, and neglect: A systematic review and meta-analysis. PLoS Med 9:e1001349.

5. Farrington DP (2005): Childhood origins of antisocial behavior. Clin Psychol Psychother 12:177-190.

6. Audrain-McGovern J, Rodriguez D, Epstein LH, Cuevas J, Rodgers K, Wileyto EP (2009): Does delay discounting play an etiological role in smoking or is it a consequence of smoking? Drug Alcohol Depend 103:99-106.

7. White JL, Moffitt TE, Caspi A, Bartusch DJ, Needles DJ, StouthamerLoeber M (1994): Measuring impulsivity and examining its relationship to delinquency. J Abnorm Psychol 103:192-205.

8. de Water E, Cillessen AH, Scheres A (2014): Distinct age-related differences in temporal discounting and risk taking in adolescents and young adults. Child Dev 85:1881-1897.

9. Steinberg $L$ (2010): A dual systems model of adolescent risk-taking. Dev Psychobiol 52:216-224.

10. Moffitt TE (1993): Adolescence-limited and life-course-persistent antisocial behavior: A developmental taxonomy. Psychol Rev 100: 674-701.

11. Jentsch JD, Taylor JR (1999): Impulsivity resulting from frontostriatal dysfunction in drug abuse: Implications for the control of behavior by reward-related stimuli. Psychopharmacology (Berl) 146:373-390.

12. Bechara A (2005): Decision making, impulse control and loss of willpower to resist drugs: A neurocognitive perspective. Nat Neurosci 8:1458-1463.

13. Gogtay N, Giedd JN, Lusk L, Hayashi KM, Greenstein D, Vaituzis AC, et al. (2004): Dynamic mapping of human cortical development during childhood through early adulthood. Proc Natl Acad Sci U S A 101: 8174-8179.

14. Sowell ER, Thompson PM, Toga AW (2004): Mapping changes in the human cortex throughout the span of life. Neuroscientist 10:372-392.

15. Casey BJ, Getz S, Galvan A (2008): The adolescent brain. Dev Rev 28: 62-77.

16. Evenden JL (1999): Varieties of impulsivity. Psychopharmacology (Berl) 146:348-361

17. Ainslie G (1975): Specious reward: A behavioral theory of impulsiveness and impulse control. Psychol Bull 82:463-496

18. Mitchell SH (1999): Measures of impulsivity in cigarette smokers and non-smokers. Psychopharmacology (Berl) 146:455-464.

19. Kirby KN, Petry NM (2004): Heroin and cocaine abusers have higher discount rates for delayed rewards than alcoholics or non-drug-using controls. Addiction 99:461-471.

20. Mackillop J, Amlung MT, Few LR, Ray LA, Sweet LH, Munafo MR (2011): Delayed reward discounting and addictive behavior: A metaanalysis. Psychopharmacology (Berl) 216:305-321.

21. Alessi SM, Petry NM (2003): Pathological gambling severity is associated with impulsivity in a delay discounting procedure. Behav Processes 64:345-354

22. Bickel WK, Jarmolowicz DP, Mueller ET, Koffarnus MN, Gatchalian KM (2012): Excessive discounting of delayed reinforcers as a transdisease process contributing to addiction and other disease-related vulnerabilities: Emerging evidence. Pharmacol Ther 134:287-297.

23. Bobova L, Finn PR, Rickert ME, Lucas J (2009): Disinhibitory psychopathology and delay discounting in alcohol dependence: Personality and cognitive correlates. Exp Clin Psychopharmacol 17: 51-61.

24. Petry NM (2002): Discounting of delayed rewards in substance abusers: Relationship to antisocial personality disorder. Psychopharmacology (Berl) 162:425-432.

25. Koffarnus MN, Jarmolowicz DP, Mueller ET, Bickel WK (2013): Changing delay discounting in the light of the competing neurobehavioral decision systems theory: A review. J Exp Anal Behav 99: $32-57$. 
26. Schumann G, Loth E, Banaschewski T, Barbot A, Barker G, Buchel C, et al. (2010): The IMAGEN study: Reinforcement-related behaviour in normal brain function and psychopathology. Mol Psychiatry 15: 1128-1139.

27. Peterson A, Crockett L, Richards M, Boxer A (1988): A self-report measure of pubertal status: Reliability, validity and initial norms. $J$ Youth Adolosc 17:117-133.

28. Wechsler D, Kaplan E, Fein D, Kramer J, Morris R, Delis D, et al. (2004): In: Wechsler Intelligence Scale for Children Fourth EditionIntegrated. San Antonio, TX: Harcourt.

29. Newcomb MD, Huba GJ, Bentler PM (1981): A multidimensional assessment of stressful life events among adolescents: Derivation and correlates. J Health Soc Behav 22:400-415.

30. Hibell B, Andersson B (1997): Alkohol-och narkotikavanor bland ungdomar i 26 länder. Alkohol och Narkotika 91:25-27.

31. Hibell B, Andersson B, Bjarnason T, Ahlström S, Balakireva O, Kokkevi A, Morgan M (2004): The ESPAD report 2003: Alcohol and other drug use among students in 35 European countries. Stockholm, Sweden: Swedish Council for Information on Alcohol and Other Drugs.

32. Olweus D (1996): The Revised Olweus Bully/Victim Questionnaire. Bergen, Norway: Research Center for Health Promotion, University of Bergen.

33. Brener ND, Collins JL, Kann L, Warren CW, Williams BI (1995): Reliability of the Youth Risk Behavior Survey Questionnaire. Am J Epidemiol 141:575-580.

34. Kirby KN (2009): One-year temporal stability of delay-discount rates. Psychon Bull Rev 16:457-462.

35. Mazur JE (1987): An adjusting procedure for studying delayed reinforcement. In: Commons ML, Mazur JE, Nevin JA, Rachlin H, editors. Quantitative Analyses of Behavior: The Effect of Delay and of Intervening Events on Reinforcement Value, vol 5 . Hillsdale, NJ: Erlbaum, 55-73.

36. Madden GJ, Begotka AM, Raiff BR, Kastern LL (2003): Delay discounting of real and hypothetical rewards. Exp Clin Psychopharmacol 11:139-145.

37. Madden GJ, Raiff BR, Lagorio CH, Begotka AM, Mueller AM, Hehli DJ, Wegener AA (2004): Delay discounting of potentially real and hypothetical rewards: II. Between- and within-subject comparisons. Exp Clin Psychopharmacol 12:251-261.

38. Kirby KN, Petry NM, Bickel WK (1999): Heroin addicts have higher discount rates for delayed rewards than non-drug-using controls. J Exp Psychol Gen 128:78-87.

39. Ashburner J, Friston KJ (2000): Voxel-based morphometry-the methods. Neuroimage 11:805-821.

40. Ashburner J (2007): A fast diffeomorphic image registration algorithm. Neuroimage 38:95-113.

41. Cox RW (1996): AFNI: Software for analysis and visualization of functional magnetic resonance neuroimages. Comput Biomed Res 29:162-173.

42. Hayes A (2009): Beyond Baron and Kenny: Statistical mediation analysis in the new millenium. Commun Monogr 74:408-420.

43. Baron RM, Kenny DA (1986): The moderator-mediator variable distinction in social psychological research: Conceptual, strategic, and statistical considerations. J Pers Soc Psychol 51:1173-1182.

44. Christakou A, Brammer M, Rubia K (2011): Maturation of limbic corticostriatal activation and connectivity associated with developmental changes in temporal discounting. Neuroimage 54:1344-1354.

45. Dalley JW, Mar AC, Economidou D, Robbins TW (2008): Neurobehavioral mechanisms of impulsivity: Fronto-striatal systems and functional neurochemistry. Pharmacol Biochem Behav 90:250-260.
46. Cardinal RN (2006): Neural systems implicated in delayed and probabilistic reinforcement. Neural Netw 19:1277-1301.

47. Wesley MJ, Bickel WK (2014): Remember the future II: Meta-analyses and functional overlap of working memory and delay discounting. Biol Psychiatry 75:435-448.

48. Plassmann H, O’Doherty JP, Rangel A (2010): Appetitive and aversive goal values are encoded in the medial orbitofrontal cortex at the time of decision making. J Neurosci 30:10799-10808.

49. Bechara A, Tranel D, Damasio H (2000): Characterization of the decision-making deficit of patients with ventromedial prefrontal cortex lesions. Brain 123:2189-2202.

50. Craig AD (2002): How do you feel? Interoception: The sense of the physiological condition of the body. Nat Rev Neurosci 3:655-666.

51. Naqvi NH, Bechara A (2009): The hidden island of addiction: The insula. Trends Neurosci 32:56-67.

52. Schwartz DL, Mitchell AD, Lahna DL, Luber HS, Huckans MS, Mitchell SH, Hoffman WF (2010): Global and local morphometric differences in recently abstinent methamphetamine-dependent individuals. Neuroimage 50:1392-1401.

53. Cho SS, Pellecchia G, Aminian K, Ray N, Segura B, Obeso I, Strafella AP (2013): Morphometric correlation of impulsivity in medial prefrontal cortex. Brain Topogr 26:479-487

54. Bjork JM, Momenan R, Hommer DW (2009): Delay discounting correlates with proportional lateral frontal cortex volumes. Biol Psychiatry 65:710-713.

55. Yu R (2012): Regional white matter volumes correlate with delay discounting. PloS One 7:e32595

56. Olson EA, Collins PF, Hooper CJ, Muetzel R, Lim KO, Luciana M (2009): White matter integrity predicts delay discounting behavior in 9to 23-year-olds: A diffusion tensor imaging study. J Cogn Neurosci 21:1406-1421.

57. Dannlowski U, Stuhrmann A, Beutelmann V, Zwanzger P, Lenzen T, Grotegerd D, et al. (2012): Limbic scars: Long-term consequences of childhood maltreatment revealed by functional and structural magnetic resonance imaging. Biol Psychiatry 71:286-293.

58. Lupien SJ, McEwen BS, Gunnar MR, Heim C (2009): Effects of stress throughout the lifespan on the brain, behaviour and cognition. Nat Rev Neurosci 10:434-445

59. Van Dam NT, Rando K, Potenza MN, Tuit K, Sinha R (2014): Childhood maltreatment, altered limbic neurobiology, and substance use relapse severity via trauma-specific reductions in limbic gray matter volume. JAMA Psychiatry 71:917-925.

60. Gorka AX, Hanson JL, Radtke SR, Hariri AR (2014): Reduced hippocampal and medial prefrontal gray matter mediate the association between reported childhood maltreatment and trait anxiety in adulthood and predict sensitivity to future life stress. Biol Mood Anxiety Disord 4:12.

61. Rao U, Chen LA, Bidesi AS, Shad MU, Thomas MA, Hammen CL (2010): Hippocampal changes associated with early-life adversity and vulnerability to depression. Biol Psychiatry 67:357-364.

62. Schilling C, Kuhn S, Romanowski A, Banaschewski T, Barbot A, Barker GJ, et al. (2013): Common structural correlates of trait impulsiveness and perceptual reasoning in adolescence. Hum Brain Mapp 34:374-383.

63. Heinz AJ, Beck A, Meyer-Lindenberg A, Sterzer P, Heinz A (2011): Cognitive and neurobiological mechanisms of alcohol-related aggres sion. Nat Rev Neurosci 12:400-413.

64. Johnson MW, Bickel WK (2002): Within-subject comparison of real and hypothetical money rewards in delay discounting. J Exp Anal Behav 77:129-146. 